\title{
Shared neural resources between left and right interlimb coordination skills: The neural substrate of abstract motor representations
}

\author{
S.P. Swinnen ${ }^{\text {a,* }}$, S. Vangheluwe ${ }^{\text {a }}$, J. Wagemans ${ }^{\text {b }}$, J.P. Coxon ${ }^{\text {a }}$, D.J. Goble ${ }^{\text {a }}$, A. Van Impe ${ }^{\text {a }}$, S. Sunaert ${ }^{\text {, }}$, \\ R. Peeters ${ }^{c}, N$. Wenderoth ${ }^{\mathrm{a}}$ \\ a Laboratory of Motor Control, Research Center for Motor Control and Neuroplasticity, Group Biomedical Sciences, K.U.Leuven, Belgium \\ b Laboratory of Experimental Psychology, Group Humanities, K.U.Leuven, Belgium \\ ' Department of Radiology, University Hospital, Group Biomedical Sciences, K.U.Leuven, Belgium
}

\section{A R T I C L E I N F O}

\section{Article history:}

Received 7 July 2009

Revised 14 August 2009

Accepted 15 October 2009

Available online 27 October 2009

\section{Keywords:}

fMRI

Interlimb coordination

Hemispheric specialization

Motor equivalence

Mirror neurons

Abstract representation

Effector independence

\begin{abstract}
A B S T R A C T
Functional magnetic resonance imaging was used to reveal the shared neural resources between movements performed with effectors of the left versus right body side. Prior to scanning, subjects extensively practiced a complex coordination pattern involving cyclical motions of the ipsilateral hand and foot according to a $90^{\circ}$ out-of-phase coordination mode. Brain activity associated with this (nonpreferred) coordination pattern was contrasted with pre-existing isodirectional (preferred) coordination to extract the learning-related brain networks. To identify the principal candidates for effector-independent movement encoding, the conjunction of training-related activity for left and right limb coordination was determined. A dominantly left-lateralized parietal-to-(pre)motor activation network was identified, with activation in inferior and superior parietal cortex extending into intraparietal sulcus and activation in the premotor areas, including inferior frontal gyrus (pars opercularis). Similar areas were previously identified during observation of complex coordination skills by expert performers. These parietal-premotor areas are principal candidates for abstract (effector-independent) movement encoding, promoting motor equivalence, and they form the highest level in the action representation hierarchy.
\end{abstract}

(c) 2009 Elsevier Inc. All rights reserved.

\section{Introduction}

Once a motor skill is acquired, it can often be performed with effectors not previously involved in practice. A typical example of this is handwriting, where the necessary strokes that were initially acquired by the dominant hand, can also be produced with the nondominant hand, foot, or other segments (Wright, 1990). This ability to accomplish the same goal by variable means is referred to as motor equivalence (Lashley, 1930) and suggests that an abstract movement representation is engraved during practice, independent of the specific muscle activations. This observation has inspired considerable research efforts into identification of the motor program's content, defined as an abstract code of motor information in memory, without specific reference to the effectors recruited for skilled performance (Schmidt, 1975).

Considerable behavioral support for limb-independent movement representations has been obtained from transfer studies evaluating the influence of learning a task with one effector on performance with another (Imamizu and Shimojo, 1995; Grafton et al., 1998; Criscimagna-Hemminger et al., 2003; Vangheluwe et al., 2004, 2005).

\footnotetext{
* Corresponding author. Research Center for Motor Control and Neuroplasticity, Tervuursevest 101, B-3001 Heverlee, Belgium. Fax: +32 16329197.

E-mail address: Stephan.swinnen@faber.kuleuven.be (S.P. Swinnen).
}

However, much less is known about their neural implementation within the central nervous system. More than hundred years ago, Liepmann elaborated upon the limb-independent neural foundation for motor skills and proposed that movement representations (i.e. "movement formula") are stored in the left parietal cortex and forwarded to bilateral frontal areas, controlling movements at either body side (Liepmann, 1905).

Examining commonly activated areas across motor skills performed with the right versus left limbs, imaging work has supported the important role of the left hemisphere in controlling movements bilaterally, generally supporting Liepmann's viewpoint (KuhtzBuschbeck et al., 2003; Haaland et al., 2004). Furthermore, studies have also explored neural representations of abstract codes for movements performed with effectors on the same body side. However, none of the aforementioned studies addressed this question in the context of acquisition of new complex coordination skills with both sides of the body. This is not a trivial matter because the search for shared neural resources and the associated lateralization of brain activity depends on many factors, such as the type of effector, task complexity, skill level, and the amount of task experience.

Here, we used fMRI to trace the neural basis of motor equivalence, inferring shared (effector-independent) neural activations across newly acquired left and right limb coordination tasks. A novel approach was used, consisting of three stages. First, to guarantee 
the formation of a new motor representation, subjects were involved in substantial practice of an unfamiliar ipsilateral coordination task, requiring cyclical flexion-extension movements of the right (or left) hand and foot, such that one segment lagged with respect to the other by a quarter of a cycle (also known as $90^{\circ}$ out-of-phase, $\Phi=90^{\circ}$ ). This new pattern differs from the preferred ipsilateral coordination mode in which both limb segments are moved in the same direction in extrinsic space (i.e., isodirectional, $\Phi=0^{\circ}$ ). Whereas the latter pattern can be produced spontaneously and with minimal effort, the acquisition of nonpreferred phase relations requires substantial practice (for bimanual coordination examples, see Lee et al., 1995; Swinnen et al., 1997a,b; Debaere et al., 2004b; Zanone and Kelso, 1992). Second, to focus on the formation of new motor representations, the activations obtained during the learned (nonpreferred) coordination mode were contrasted against those of the isodirectional (preferred) mode. Third, the regions that were commonly active across the learned left and right limb coordination modes were identified by means of a conjunction analysis.

This procedure allowed us to identify critical candidate areas supporting the abstract (effector-independent) features of the central representation, developed during skill acquisition. Although previous studies have compared brain activations associated with left and right limb movements, use of a learned complex coordination pattern involving upper and lower limb segments has not been made, nor has the aforementioned three-stage analysis approach been implemented. Using this new approach, brain areas within the parieto-frontal network were targeted, as identified by Liepmann's seminal work and subsequent ideomotor apraxia studies (Haaland et al., 2000). Furthermore, based on recent action observation studies of complex gross motor skills (Calvo-Merino et al., 2005; Cross et al., 2006), we anticipated that the superior and inferior parietal lobe and the inferior frontal gyrus, would play a prominent role in the abstract spatiotemporal codes for complex coordination skills.

\section{Materials and methods}

\section{Subjects}

Fourteen subjects (eight males and six females, age 19-28 years) participated in the experiment. They were all right-handed, as assessed by the Edinburgh Handedness Inventory (Oldfield, 1971) and naive with respect to the task. Prior to participation, subjects were informed about the experimental procedure and provided written informed consent. The study design was approved by the local Ethics Committee of Biomedical Research at K.U. Leuven and was in accordance with the guidelines of the Declaration of Helsinki (1964).

\section{Experimental design}

Task

Subjects acquired a left/right cyclical ipsilateral hand-foot coordination task, requiring rhythmical flexion and extension movements, with one limb required to lead the other by a quarter cycle, resulting in a phase offset of $90^{\circ}\left(90^{\circ}\right.$ TASK). This task can only be performed after substantial practice, implying that a novel representation of the task has to be acquired (for bimanual examples, see Lee et al., 1995; Zanone and Kelso, 1992). By contrast, isodirectional coordination, in which both limb segments are moved upwards and downwards simultaneously (ISO task), is inherent to our motor system and does not require practice for successful performance (Baldissera et al., 1982; Carson et al., 1995; Swinnen et al., 1995). Preferred coordination modes do not exhibit substantial changes in brain activation when practiced extensively (for a preferred bimanual pattern, see Debaere et al., 2004a). Moreover, preferred (isodirectional) movements produce the same net motor output (i.e., flexion-extension of the same effectors), but with a different spatio-temporal pattern.
Accordingly, it is an optimal control condition to ensure equality of kinematics. All movements were metronome paced (KORG DTM-12, Tokyo, Japan) at a movement frequency of $1.1 \mathrm{~Hz}$ (66 beats per minute), whereby an entire movement cycle had to be completed on every beat. The coordination tasks were performed at the right ( $r$ ) and left (l) body side. Compared with the sequencing and adaptation tasks predominantly used in medical imaging studies on learning, the present coordination task combines successive as well as simultaneous motor elements into an integrated complex spatiotemporal organizational structure, i.e., directional relations between the limb segments change at each quarter cycle. Moreover, the principles governing interlimb coordination are not a mere extrapolation of those involved in unimanual tasks (Swinnen, 2002).

\section{Kinematic registration}

Laying supine inside the actual or "dummy" scanner, subjects' forearms and lower legs were supported by a cushion to facilitate free wrist and ankle rotation. The limb segments were positioned in nonferromagnetic wrist-hand and ankle-foot orthoses, restricting the movements to flexion-extension in the sagittal plane. The anatomical joint axis was aligned with the axis of rotation of the orthosis. High precision shaft encoders (4096 pulses per revolution, sampling frequency $100 \mathrm{~Hz}$ ) were fixed to the frictionless movement axis of each orthosis to register angular displacements of the joints on-line during both training and scanning.

\section{Training}

Before scanning, the $90^{\circ}$ out-of-phase pattern was intensively practiced during five successive days. At the start of each daily learning session, subjects performed five trials (30 s per trial) of the isodirectional coordination task $\left(\Phi=0^{\circ}\right)$ with their right and left body side. Then, they practiced the $90^{\circ}$ out-of-phase coordination task for 60 trials per day, subdivided into twelve blocks of five trials ( $30 \mathrm{~s}$ per trial), amounting to $>8000$ individual movement cycles across the 5 days of practice. Organization of training, using the left or right body side, was randomized across subjects. Participants switched to a different body side following every three practice blocks. During learning, augmented visual feedback was provided by means of a Lissajous figure, which represented the wrist movement on the $X$-axis and the ankle movement on the $Y$-axis. A circle appeared on the screen when participants correctly performed the $90^{\circ}$ out-of-phase coordination task, providing them with on-line feedback in real time to support performance. To learn to generate the task internally (i.e., without any visual feedback), the number of visually guided trials was gradually decreased across the 5 learning days, i.e. from five to two feedback trials per block across days 2 to 5 (fading feedback schedule).

\section{Scanning}

The general experimental setup was the same as in the dummy scanner. Participants performed the following conditions in the scanner: (1) the learned $90^{\circ}$ out-of-phase task with their left $\left(90^{\circ} 1\right)$ and (2) right $\left(90^{\circ} \mathrm{r}\right)$ body side, (3) the isodirectional task with their left (ISO l) and (4) right (ISO r) body side, and (5) a rest condition in which no movements were performed (rest). Movement imagery conditions were also included but these are not reported here. The condition order was randomized across subjects. All conditions were performed without visual feedback and were metronome paced. To avoid confounding eye movements, participants were trained to fixate a cross, displayed in front of them. Head movements were restricted by a bite-bar. The mean translation and rotation parameters for the whole group of subjects were $0.36,0.54,0.80$ and $0.47,0.40$. and 0.34 , respectively.

Following training, task related changes in neural activity were determined by measuring the blood oxygen level-dependent (BOLD) signal using echoplanar imaging on a $3 \mathrm{~T}$ Intera MR scanner (Philips, Best, The Netherlands), with a 6-element SENSE head coil (MRI 
Devices Corp., Waukesha, WI). Each scan session began with the acquisition of a 3D SENSE high resolution T1-weighted image $(\mathrm{TR} / \mathrm{TE}=9.68 / 4.6 \mathrm{~ms}, \mathrm{TI}=1100 \mathrm{~ms}$, field of view $=250 \mathrm{~mm}$, matrix $=256 \times 256$, slice thickness $=1.2 \mathrm{~mm}, 182$ slices, SENSE factor $=2$ ) for anatomical detail. Then, participants performed eight runs, each consisting of 140 whole brain gradient-echo echoplanar T2-weighted functional images $(\mathrm{TR} / \mathrm{TE}=3000 / 33 \mathrm{~ms}$, field of view $=$ $230 \mathrm{~mm}$, matrix $=112 \times 112$, slice thickness $=4.0 \mathrm{~mm}, 34$ sagittal slices, SENSE factor $=2$ ). Each run contained two blocks of the following conditions: (1) $90^{\circ} 1$, (2) $90^{\circ} \mathrm{r}$, (3) iso l, (4) iso r, and (5) rest. Each condition lasted $21 \mathrm{~s}$ (corresponding to seven whole brain images) and was triggered by a visual template symbolizing the task to be performed for $3 \mathrm{~s}$. The order of conditions was randomized across runs and subjects.

\section{Data analyses}

\section{Kinematic analysis}

To determine the quality of the produced coordination pattern, we first calculated the continuous phase angle $\theta$ for each limb, using the following formula: $\theta_{\mathrm{w} / \mathrm{a}}=\tan ^{-1}\left[\left(\mathrm{~d} X_{\mathrm{w} / \mathrm{a}} / \mathrm{d} t\right) / X_{\mathrm{w} / \mathrm{a}}\right]$, whereby $X$ describes the normalized displacement of the limb (after rescaling to the interval $[-1,1]$ for each cycle of oscillation) and $\mathrm{d} X / \mathrm{d} t$ is its normalized instantaneous velocity (adapted from Kelso et al., 1986). The indices $\mathrm{w}$ and a represent the wrist and ankle joint, respectively. The relative phase was calculated by subtracting the phase angles of both limbs, $\Phi=\theta_{\mathrm{w}}-\theta_{\mathrm{a}}$. Absolute deviations from the target relative phase (i.e. $0^{\circ}$ and $90^{\circ}$ for the isodirectional and $90^{\circ}$ out-of-phase coordination pattern, respectively) were calculated as a measure of coordination accuracy (AEФ).

Repeated measures analyses were conducted on the training trials without visual feedback to reveal learning of the $90^{\circ}$ out-of-phase pattern. This was also the condition examined in the scanner. The first analysis addressed the performance changes (AEФ) of the $90^{\circ}$ out-ofphase pattern across the last four practice days, involving a $2 \times 2 \times 4$ Body side (right, left $) \times$ Coordination Mode $\left(90^{\circ}\right.$ task, ISO task $) \times$ Day (days 2 to 5 ) repeated measures ANOVA. The analysis was restricted to days 2-5 because practice day 1 contained no trials without augmented visual feedback. Significant interactions were further examined by a Tukey post-hoc test. The level of significance was set to $\alpha=0.05$.

An additional analysis verified whether a performance plateau on the $90^{\circ}$ out-of-phase pattern was reached across the 6 training blocks of practice day 5 and whether it persisted during the scanning session. For that purpose, we performed a $2 \times 7$ (Body Side $\times$ Blocks) ANOVA with repeated measures on both factors. Body side consisted of the left and right $90^{\circ}$ out-of-phase pattern and Blocks consisted of 7 levels, i.e., the 6 blocks of practice day 5 and 1 scanning block. We were particularly interested in testing the Body side $\times$ Time interaction.

In addition, mean cycle duration and amplitude were computed for the hand and foot kinematics obtained during scanning. Mean cycle duration was defined as the average duration across individual movement cycles within a trial. Movement amplitude consisted of the absolute peak-to-peak amplitude for wrist and foot, averaged across individual movement cycles. A $2 \times 2 \times 2$ Body Side (right, left) $\times$ Limb Segment (hand, foot) $\times$ Coordination Mode $\left(90^{\circ}\right.$ task, ISO task) ANOVA with repeated measures on all factors was performed.

\section{Imaging analysis}

Imaging data were analyzed with the Statistical Parametric Mapping software (SPM2, Wellcome Department of Imaging Neuroscience, London, UK, http:// www.fil.ion.ucl.ac.uk). Functional images were realigned to the first volume of the first run. A mean image of the realigned volumes was created, subsequently smoothed with a Gaussian kernel of $6 \mathrm{~mm}$ full width at half maximum (FWHM) and co-registered to the anatomical T1-weighted image. To normalize the anatomical image and the EPI's to a standard reference frame (Talairach and Tournoux, 1998), the anatomical as well as representative template image (MNI, Montreal Neurological Institute) were first segmented into gray matter, white matter and cerebrospinal fluids. After smoothing the anatomical gray matter image $(6 \mathrm{~mm}$ FWHM), it was normalized to the gray matter of the MNI brain. Finally, the derived normalization parameters were applied to the EPI images, which were subsampled to a voxel size of $2 \times 2 \times 2 \mathrm{~mm}$ and smoothed with a Gaussian kernel of $8 \mathrm{~mm}$ FWHM.

For the first level analysis, a general linear model was used, containing for each condition a boxcar function convolved with the SPM2 haemodynamic response function. An appropriate high-pass filter was applied to remove low frequency drifts. Six movement parameters derived from realignment, were included as covariates of no interest to correct for head movements. Contrasts of interest were calculated for each subject individually (averaged across runs) and subsequently entered into a second-level random effects analysis. A series of different contrasts were computed in which we gradually focused on those areas that were shared between the learned movement with the effectors of the left and right body side, i.e., representing the $90^{\circ}$ out-of-phase movement at the more abstract representational level.

First, each coordination task was contrasted with rest to reveal the general brain network activated during the different coordination patterns $(p<0.001$ corrected for multiple comparisons by means of the false discovery rate, FDR).

Second, to identify those areas representing new coordination learning as compared to performance of pre-existing coordination modes, we determined the brain areas that were significantly more involved during the $90^{\circ}$ out-of-phase than intrinsic isodirectional coordination pattern, resulting in the following contrasts: $90^{\circ} \mathrm{r}-\mathrm{ISO} \mathrm{r}$ and $90^{\circ} 1-$ ISO 1 . To subsequently reveal which areas were commonly activated while executing the $90^{\circ}$ versus iso task, independent of the moving limbs, the following conjunction was calculated: $\left(90^{\circ} \mathrm{r}-\mathrm{ISO} \mathrm{r}\right) \cap$ ( $90^{\circ}$ l-ISO 1) (Nichols et al., 2005). The regions thus identified are possible candidates for the effector-independent neural correlate of the $90^{\circ}$ out-of-phase pattern. Correction for multiple comparisons was applied by means of a false discovery rate (FDR) procedure at $p<0.01$, resulting in a t-threshold of $t>3.49$ (Genovese et al., 2002). Minimum cluster size was set at 20 voxels.

\section{Results}

\section{Kinematic data}

As expected, the Body Side $\times$ Coordination Mode $\times$ Day ANOVA on the absolute error of relative phase (AEФ) obtained during training demonstrated that the relative phase for the ISO task remained constant, whereas the left and right $90^{\circ}$ coordination tasks improved across days of practice (i.e., errors decreased) (Fig. 1). This was

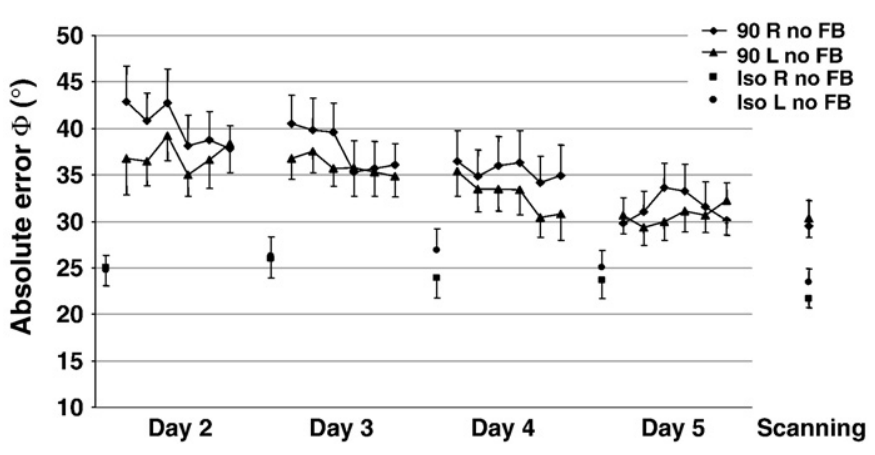

Fig. 1. Absolute error scores of relative phase across learning days and during scanning Standard errors are denoted by error bars. 
Table 1

Brain areas activated during ipsilateral coordination tasks versus rest.

\begin{tabular}{|c|c|c|c|c|c|c|c|c|c|c|c|c|c|c|c|c|c|}
\hline \multirow[t]{3}{*}{ Brain region } & \multirow[t]{3}{*}{ Hemisphere } & \multicolumn{4}{|c|}{ ISO R-rest } & \multicolumn{4}{|c|}{ ISO L-rest } & \multicolumn{4}{|c|}{$90^{\circ} \mathrm{R}$-rest } & \multicolumn{4}{|c|}{$90^{\circ} \mathrm{L}$-rest } \\
\hline & & \multicolumn{3}{|c|}{ Coordinates } & \multirow[t]{2}{*}{$t$-value } & \multicolumn{3}{|c|}{ Coordinates } & \multirow[t]{2}{*}{$t$-value } & \multicolumn{3}{|c|}{ Coordinates } & \multirow[t]{2}{*}{$t$-value } & \multicolumn{3}{|c|}{ Coordinates } & \multirow[t]{2}{*}{$t$-value } \\
\hline & & $x$ & $y$ & $z$ & & $x$ & $y$ & $z$ & & $x$ & $y$ & $z$ & & $x$ & $y$ & $z$ & \\
\hline \multirow[t]{2}{*}{ Superior frontal gyrus (SMA) } & $\mathrm{L}$ & -12 & -14 & 58 & 8.00 & - & - & - & - & -4 & -18 & 58 & 8.81 & -4 & -4 & 70 & 8.16 \\
\hline & $\mathrm{R}$ & 8 & -8 & 62 & 8.15 & 14 & -18 & 68 & 13.09 & 8 & -8 & 62 & 9.56 & 10 & -10 & 62 & 9.22 \\
\hline \multirow[t]{2}{*}{ Superior frontal sulcus (PMd) } & $\mathrm{L}$ & - & - & - & - & - & - & - & - & -28 & -14 & 60 & 9.09 & - & - & - & - \\
\hline & $\mathrm{R}$ & - & - & - & - & - & - & - & - & - & - & - & - & 30 & -18 & 60 & 9.45 \\
\hline \multirow[t]{2}{*}{ Precentral gyrus (PMd) } & $\mathrm{L}$ & -34 & -26 & 58 & 9.21 & - & - & - & - & -34 & -28 & 58 & 10.26 & -20 & -12 & 72 & 5.78 \\
\hline & $\mathrm{R}$ & - & - & - & - & 28 & -18 & 58 & 8.85 & - & - & - & - & 14 & -20 & 66 & 13.25 \\
\hline \multirow[t]{2}{*}{ Central sulcus } & $\mathrm{L}$ & -20 & -26 & 74 & 9.23 & - & - & - & - & -16 & -18 & 70 & 10.49 & -16 & -18 & 66 & 6.47 \\
\hline & $\mathrm{R}$ & - & - & - & - & 44 & -18 & 54 & 9.30 & - & - & - & - & 36 & -30 & 62 & 9.50 \\
\hline \multirow[t]{2}{*}{ Postcentral gyrus (SM1 hand) } & $\mathrm{L}$ & -24 & -38 & 74 & 12.23 & - & - & - & - & -26 & -38 & 72 & 11.26 & - & - & - & - \\
\hline & $\mathrm{R}$ & - & - & - & - & 30 & -40 & 68 & 6.78 & - & - & - & - & 12 & -48 & 70 & 9.02 \\
\hline Paracentral lobule (SM1 foot) & $\mathrm{L}$ & -4 & -32 & 72 & 11.59 & - & - & - & - & -4 & -32 & 70 & 13.29 & - & - & - & - \\
\hline & $\mathrm{R}$ & - & - & - & - & 8 & -38 & 68 & 8.53 & - & - & - & - & 8 & -30 & 62 & 12.42 \\
\hline Cingulate sulcus & $\mathrm{L}$ & -6 & -16 & 54 & 8.38 & - & - & - & - & - & - & - & - & - & - & - & - \\
\hline & $\mathrm{R}$ & - & - & - & - & 12 & -28 & 52 & 9.33 & - & - & - & - & 8 & -4 & 50 & 7.27 \\
\hline $\begin{array}{l}\text { Superior parietal lobule/ } \\
\text { postcentral sulcus }\end{array}$ & $\mathrm{L}$ & -28 & -40 & 72 & 11.57 & - & - & - & - & -24 & -36 & 62 & 9.99 & - & - & - & - \\
\hline Supramarginal gyrus & $\mathrm{L}$ & -54 & -26 & 20 & 8.58 & - & - & - & - & -52 & -26 & 20 & 9.25 & -54 & -28 & 20 & 9.47 \\
\hline & $\mathrm{R}$ & - & - & - & - & - & - & - & - & - & - & - & - & - & - & - & - \\
\hline Superior temporal gyrus & $\mathrm{R}$ & - & - & - & - & - & - & - & - & - & - & - & - & 56 & -40 & 26 & 8.65 \\
\hline $\begin{array}{l}\text { Posterior fundus of the lateral } \\
\text { fissure / superior temporal gyrus }\end{array}$ & $\mathrm{L}$ & -64 & -22 & 14 & 8.64 & - & - & - & - & -62 & -22 & 14 & 7.71 & -62 & -22 & 14 & 7.10 \\
\hline & $\mathrm{R}$ & - & - & - & - & 40 & -36 & 12 & 8.43 & - & - & - & - & 52 & -26 & 14 & 10.22 \\
\hline Insula & $\mathrm{L}$ & - & - & - & - & - & - & - & - & -34 & -24 & 14 & 8.32 & -48 & 4 & 2 & 6.58 \\
\hline & $\mathrm{R}$ & - & - & - & - & - & - & - & - & - & - & - & - & 46 & 2 & 6 & 8.84 \\
\hline Putamen & $\mathrm{L}$ & -34 & -14 & 4 & 13.88 & - & - & - & - & -24 & -8 & 8 & 10.62 & -24 & -2 & 8 & 7.29 \\
\hline & $\mathrm{R}$ & - & - & - & - & 30 & -10 & 2 & 7.70 & - & - & - & - & 30 & -8 & 4 & 10.73 \\
\hline Globus pallidus & $\mathrm{L}$ & -22 & -4 & -4 & 7.93 & - & - & - & - & -20 & -12 & -2 & 7.33 & -18 & -2 & 0 & 6.56 \\
\hline & $\mathrm{R}$ & - & - & - & - & & & & & - & - & - & - & 18 & 2 & 2 & 7.23 \\
\hline Thalamus & $\mathrm{L}$ & -20 & -8 & -14 & 9.09 & - & - & - & - & -16 & -22 & 0 & 7.04 & -20 & -12 & 18 & 6.86 \\
\hline & $\mathrm{R}$ & - & - & - & - & 22 & -18 & 4 & 6.48 & - & - & - & - & 12 & -24 & -2 & 6.20 \\
\hline Cerebellar vermis (IV) & $\mathrm{L}$ & - & - & - & - & - & - & - & - & - & - & - & - & -6 & -50 & -16 & 9.89 \\
\hline & $\mathrm{R}$ & 14 & -48 & -24 & 12.51 & - & - & - & - & 14 & -50 & -24 & 12.22 & 6 & -48 & -26 & 7.03 \\
\hline Cerebellar vermis (V) & $\mathrm{L}$ & - & - & - & - & -6 & -62 & -16 & 11.36 & - & - & - & - & -8 & -64 & -18 & 10.23 \\
\hline & $\mathrm{R}$ & 8 & -62 & -18 & 19.16 & - & - & - & - & 8 & -62 & -18 & 17.11 & - & - & - & - \\
\hline Cerebellar hemisphere (V) & $\mathrm{L}$ & - & - & - & - & - & - & - & - & - & - & - & - & -24 & -44 & -28 & 10.50 \\
\hline & $\mathrm{R}$ & - & - & - & - & - & - & - & - & - & - & - & - & - & - & - & - \\
\hline Cerebellar hemisphere (VI) & $\mathrm{L}$ & - & - & - & - & -28 & -52 & -26 & 10.49 & - & - & - & - & -26 & -52 & -26 & 10.33 \\
\hline & $\mathrm{R}$ & - & - & - & - & - & - & - & - & 30 & -46 & -34 & 7.95 & 28 & -48 & -32 & 5.59 \\
\hline Cerebellar hemisphere (VIII A) & $\mathrm{L}$ & - & - & - & - & -26 & -58 & -52 & 8.67 & - & - & - & - & -18 & -68 & -54 & 8.81 \\
\hline & $\mathrm{R}$ & 26 & -58 & -50 & 8.16 & - & - & - & - & 26 & -56 & -50 & 12.24 & 30 & -64 & -54 & 7.68 \\
\hline Cerebellar hemisphere (VIII B) & $\mathrm{L}$ & - & - & - & - & -16 & -52 & -60 & 8.13 & - & - & - & - & -26 & -50 & -50 & 10.87 \\
\hline & $\mathrm{R}$ & 14 & -58 & -58 & 9.46 & - & - & - & - & 26 & -50 & -52 & 11.84 & - & - & - & - \\
\hline
\end{tabular}


confirmed by a significant Coordination Mode $\times$ Day interaction $(p<0.01)$. Post-hoc tests revealed that performance on the intrinsic ISO task was initially significantly better than the $90^{\circ}$ task $(p<0.001)$ and exhibited no changes across learning days ( $p>0.99$ ), whereas the practiced $90^{\circ}$ pattern improved significantly across days $(p<0.05)$. A comparable performance evolution was obtained for the right and left body side, as inferred from lack of a significant Coordination Mode $\times$ Body Side $\times$ Time interaction $(p=0.77)$. Moreover, no significant differences were obtained between the left and right $90^{\circ}$ task $(p=0.79)$.

To examine whether a performance plateau was reached, the $2 \times 7$ (Body Side $\times$ Blocks) ANOVA on the AEФ scores revealed no significant differences among the 6 blocks of the final practice day and the scanning block $(p>0.20)$. This indicated that performance of the $90^{\circ}$ coordination task reached a stable plateau during the final training day, which was preserved during scanning.

Separate $2 \times 2 \times 2$ (Body Side $\times$ Limb Segment $\times$ Coordination Mode) ANOVAs were conducted on cycle duration and amplitude measures of movements performed during scanning. Analysis of cycle duration revealed no significant main or interaction effects ( $p>0.19)$. Means were: left ISO hand and foot, 876 and $882 \mathrm{~ms}$; right ISO hand and foot, 886 and $884 \mathrm{~ms}$; left $90^{\circ}$ task hand and foot, 883 and $883 \mathrm{~ms}$; right $90^{\circ}$ task hand and foot, 883 , and $885 \mathrm{~ms}$. The analysis of amplitude revealed a significant main effect of coordination mode $(p<0.05)$. The remaining main or interaction effects were not significant $(p>0.2)$. Means were: left ISO hand and foot, 19 and $20,6^{\circ}$; right ISO hand and foot, 18,7 and $21,6^{\circ}$; left $90^{\circ}$ task hand and foot, 23,6 and $25,1^{\circ}$; right $90^{\circ}$ task hand and foot, 23,1 and $25,3^{\circ}$.

Table 2

Brain areas activated during the $90^{\circ}$ out-of-phase versus iso task.

\begin{tabular}{|c|c|c|c|c|c|c|c|c|c|}
\hline \multirow[t]{3}{*}{ Brain region } & \multirow[t]{3}{*}{ Hemisphere } & \multicolumn{4}{|c|}{$90^{\circ} \mathrm{R}>\mathrm{ISO} \mathrm{R}$} & \multicolumn{4}{|c|}{$90^{\circ} \mathrm{L}>\mathrm{ISO} \mathrm{L}$} \\
\hline & & \multicolumn{3}{|c|}{ Coordinates } & \multirow[t]{2}{*}{$t$-value } & \multicolumn{3}{|c|}{ Coordinates } & \multirow[t]{2}{*}{$t$-value } \\
\hline & & $x$ & $y$ & $z$ & & $x$ & $y$ & $z$ & \\
\hline \multirow[t]{2}{*}{ Superior frontal gyrus (SMA) } & $\mathrm{L}$ & -14 & -6 & 60 & 5.84 & -14 & -4 & 58 & 7.16 \\
\hline & $\mathrm{R}$ & 6 & -8 & 64 & 5.67 & 6 & -8 & 64 & 6.88 \\
\hline \multirow[t]{2}{*}{ Superior frontal gyrus (PMd) } & $\mathrm{L}$ & -24 & -10 & 60 & 7.31 & - & - & - & - \\
\hline & $\mathrm{R}$ & 24 & -8 & 58 & 6.70 & 28 & -10 & 58 & 6.78 \\
\hline Superior frontal sulcus (PMd) & $\mathrm{L}$ & -28 & -10 & 68 & 7.27 & -30 & -8 & 62 & 7.47 \\
\hline \multirow[t]{2}{*}{ Middle frontal gyrus (DLPFC) } & $\mathrm{L}$ & - & - & - & - & -36 & 50 & 14 & 4,82 \\
\hline & $\mathrm{R}$ & - & - & - & - & 40 & 44 & 24 & 4,40 \\
\hline \multirow[t]{2}{*}{ Inferior frontal gyrus pars opercularis } & $\mathrm{L}$ & -56 & 8 & 18 & 4.18 & -54 & 12 & 8 & 4.41 \\
\hline & $\mathrm{R}$ & 54 & 8 & 12 & 3.85 & 60 & 10 & 10 & 5.39 \\
\hline \multirow[t]{2}{*}{ Precentral gyrus (PMd) } & $\mathrm{L}$ & -18 & -10 & 72 & 7.41 & -18 & -12 & 72 & 7.47 \\
\hline & $\mathrm{R}$ & 36 & -4 & 32 & 3.43 & 22 & -16 & 68 & 6.88 \\
\hline \multirow[t]{2}{*}{ Inferior precentral sulcus (PMv) } & $\mathrm{L}$ & -58 & 6 & 26 & 4.25 & -56 & 4 & 26 & 5.98 \\
\hline & $\mathrm{R}$ & 56 & 6 & 22 & 3.54 & 58 & 8 & 12 & 5.34 \\
\hline Central sulcus & $\mathrm{L}$ & -40 & -26 & 58 & 7.95 & -14 & -38 & 74 & 4.94 \\
\hline \multirow[t]{2}{*}{ Postcentral gyrus (S1/M1 hand area) } & $\mathrm{L}$ & -48 & -30 & 54 & 7.52 & -38 & -16 & 42 & 5.59 \\
\hline & $\mathrm{R}$ & - & - & - & - & 20 & -36 & 68 & 5.34 \\
\hline Inferior postcentral sulcus & $\mathrm{L}$ & -58 & -20 & 32 & 4.66 & -44 & -22 & 38 & 4.53 \\
\hline \multirow[t]{2}{*}{ Paracentral lobule (S1/M1 foot area) } & $\mathrm{L}$ & - & - & - & - & -2 & -22 & 64 & 7.16 \\
\hline & $\mathrm{R}$ & - & - & - & - & 8 & -30 & 64 & 6.50 \\
\hline \multirow[t]{2}{*}{ Cingulate gyrus } & $\mathrm{L}$ & -18 & 8 & 40 & 6.07 & -14 & 14 & 30 & 4.77 \\
\hline & $\mathrm{R}$ & 14 & 4 & 42 & 3.71 & 6 & 6 & 40 & 4.47 \\
\hline Superior parietal gyrus & $\mathrm{L}$ & -26 & -44 & 70 & 7.64 & -38 & -46 & 60 & 7.91 \\
\hline & $\mathrm{R}$ & 24 & -48 & 70 & 4.17 & 22 & -48 & 68 & 6.55 \\
\hline Supramarginal gyrus & $\mathrm{L}$ & -58 & -28 & 22 & 3.63 & -60 & -40 & 26 & 4,76 \\
\hline & $\mathrm{R}$ & 62 & -40 & 28 & 3.29 & 48 & -24 & 34 & 3.85 \\
\hline Posterior intraparietal sulcus & $\mathrm{R}$ & 32 & -54 & 42 & 3.82 & 30 & -52 & 40 & 5.42 \\
\hline Superior parietal lobe/postcentral sulcus & $\mathrm{L}$ & -38 & -44 & 62 & 7.83 & -24 & -60 & 64 & 7.28 \\
\hline & $\mathrm{R}$ & - & - & - & - & 32 & -44 & 60 & 6.21 \\
\hline Insula & $\mathrm{L}$ & -32 & 18 & 8 & 4.11 & -32 & 16 & 6 & 5.84 \\
\hline & $\mathrm{R}$ & 34 & 18 & 6 & 3.42 & - & - & - & - \\
\hline Superior temporal gyrus & $\mathrm{L}$ & - & - & - & - & -42 & -44 & 10 & 4,54 \\
\hline & $\mathrm{R}$ & - & - & - & - & 52 & -44 & 14 & 4,14 \\
\hline Inferior temporal gyrus & $\mathrm{L}$ & - & - & - & - & -56 & -56 & 4 & 3,99 \\
\hline & $\mathrm{R}$ & - & - & - & - & 46 & -52 & -6 & 4,25 \\
\hline Putamen & $\mathrm{L}$ & -22 & 2 & 6 & 3.82 & -34 & 8 & 0 & 6.11 \\
\hline & $\mathrm{R}$ & - & - & - & - & 34 & 14 & 0 & 4.03 \\
\hline Globus pallidus & $\mathrm{L}$ & - & - & - & - & -24 & -16 & 4 & 6.45 \\
\hline & $\mathrm{R}$ & - & - & - & - & 20 & -12 & 10 & 6.44 \\
\hline Thalamus & $\mathrm{L}$ & -22 & -18 & 4 & 3.75 & -12 & -28 & 10 & 4.85 \\
\hline & $\mathrm{R}$ & - & - & - & - & 16 & -6 & 2 & 6.13 \\
\hline Cerebellar vermis (IV) & $\mathrm{R}$ & 10 & -52 & -24 & 5.97 & 4 & -52 & -22 & 6.98 \\
\hline Cerebellar vermis (V) & $\mathrm{R}$ & 4 & -56 & -18 & 6.26 & 2 & -56 & -18 & 6.97 \\
\hline Cerebellar vermis (X) & $\mathrm{R}$ & - & - & - & - & 6 & -48 & -24 & 7.10 \\
\hline Cerebellar hemisphere (V) & $\mathrm{R}$ & 24 & -44 & -32 & 5.65 & 26 & -44 & -34 & 8.54 \\
\hline Cerebellar hemisphere (VI) & $\mathrm{L}$ & - & - & - & - & -32 & -54 & -30 & 8.35 \\
\hline & $\mathrm{R}$ & 24 & -54 & -30 & 6.37 & 24 & -66 & -30 & 7.16 \\
\hline Cerebellar hemisphere (VIII A) & $\mathrm{L}$ & -30 & -62 & -52 & 4.36 & -32 & -52 & -48 & 6.86 \\
\hline & $\mathrm{R}$ & 24 & -60 & -52 & 5.05 & 30 & -68 & -54 & 6.88 \\
\hline Cerebellar hemisphere (VIII B) & $\mathrm{L}$ & - & - & - & - & -28 & -58 & -54 & 6.46 \\
\hline & $\mathrm{R}$ & - & - & - & - & 24 & -45 & -48 & 6.22 \\
\hline Cerebellar hemisphere ( $\mathrm{Cr} \mathrm{I}$ ) & $\mathrm{L}$ & -38 & -52 & -34 & 5.05 & -38 & -54 & -34 & 8.30 \\
\hline
\end{tabular}

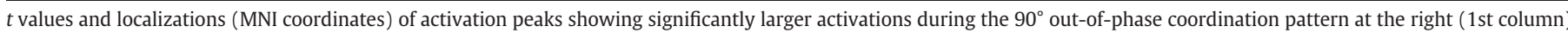

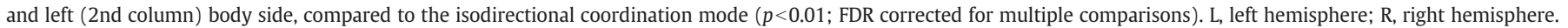


Imaging data

\section{Activations during ipsilateral coordination: ISO and $90^{\circ}$ coordination} tasks versus rest

For both the (preferred) ISO 1 and iso $\mathrm{r}$ coordination patterns, we identified a typical network for motor coordination, including the contralateral primary sensorimotor hand and foot areas (SM1), dorsal premotor area (PMd, precentral gyrus), supplementary motor area (SMA) (bilateral for ISO R), a region at the border of the superior temporal gyrus and the fundus of the lateral fissure, corresponding to the secondary somatosensory area (S2), posterior cingulate sulcus, basal ganglia, and ipsilateral anterior and posterior cerebellum (Table 1). In general, the pre-existing preferred coordination mode (ISO) was primarily associated with contralateral cortical activation.

Performance of the learned $90^{\circ}$ out-of-phase coordination pattern (nonpreferred) resulted in partly similar activations as for the ISO task (see Table 1). However, activation appeared higher and additional (sub)regions were recruited, including the contralateral superior frontal sulcus (PMd), the thalamus and the ipsilateral cerebellar hemisphere (lobule VI). Moreover, performing the left $90^{\circ}$ task additionally activated the ipsilateral superior frontal sulcus (PMd), central sulcus (i.e. SM1), insula, supramarginal gyrus, S2, basal ganglia, thalamus, and the contralateral anterior and posterior cerebellum. Accordingly, the left $90^{\circ}$ task appeared to induce more bilateral activations than the preferred ISO L task as well as both right limb tasks. The differences between the newly acquired and the (preexisting) preferred coordination modes were studied in further detail by direct contrasts, as described next.

\section{Activations significantly larger during the $90^{\circ}$ than ISO task}

To identify brain areas associated with the learned $90^{\circ}$ coordination pattern, we determined the regions exhibiting larger activation during the $90^{\circ}$ than iso task for the right and left body side, respectively (Table 2, Fig. 2). Brain regions exhibiting larger activation during the right $90^{\circ}$ versus ISO R task were the bilateral SMA, PMd (i.e. the precentral gyrus and superior frontal gyrus/sulcus), ventral premotor cortex (PMv) (i.e. the inferior precentral sulcus), rostral cingulate gyrus, superior parietal gyrus, supramarginal gyrus, insula, inferior frontal gyrus pars opercularis (IFGpo), and anterior and posterior cerebellum (all bilateral). On the contralateral (left) side, the central sulcus, (inferior) postcentral gyrus/sulcus, putamen and thalamus were activated, and, on the ipsilateral side, the cerebellar vermis (Fig. 2A, yellow).

Performing the $90^{\circ}$ versus ISO task with the left body side evoked more widespread and more bilateral activation of the same areas, extending to additional brain regions: the prefrontal cortex, postcentral sulcus/gyrus, paracentral lobule (corresponding to the SM1 foot area), superior and inferior temporal gyrus, basal ganglia, thalamus and cerebellar hemisphere (VIIIb) (all bilateral) (Table 2, Fig. 2B).

Activation common to the newly acquired left and right $90^{\circ}$ coordination task

The cerebral structures that were commonly involved in the $90^{\circ}$ out-of-phase task, independent of the recruited limb segments, were defined by means of a conjunction $\left[\left(90^{\circ} \mathrm{r}-\mathrm{ISO} \mathrm{r}\right) \cap\left(90^{\circ} \mathrm{l}-\mathrm{ISO} \mathrm{l}\right)\right]$ (Fig. 2C, Table 3). The obtained cortical regions were: bilateral PMd, SMA (Fig. 3F), IFGpo (Fig. 3A), PMv, and superior parietal cortex extending into the left intraparietal sulcus, central sulcus, postcentral gyrus (corresponding to the S1 hand area), inferior postcentral sulcus, paracentral lobule (corresponding to the S1 foot area) (Figs. 3D, E), supramarginal gyrus (Fig. 3D), insula (Fig. 3B). Subcortically, activation was observed in the right vermis of the anterior cerebellum (IV, V) and right and left hemisphere of the posterior cerebellum (right VI, left and right VIIIA) (Figs. 3F, H). Activation was also observed in left basal ganglia (putamen) and left thalamus (Figs. 3B, G). Thus, the conjunction across the left and right $90^{\circ}$ out-of-phase task resulted in a distributed cortico-subcortical network with a dominantly leftlateralized parietal-(pre)motor activation pattern.

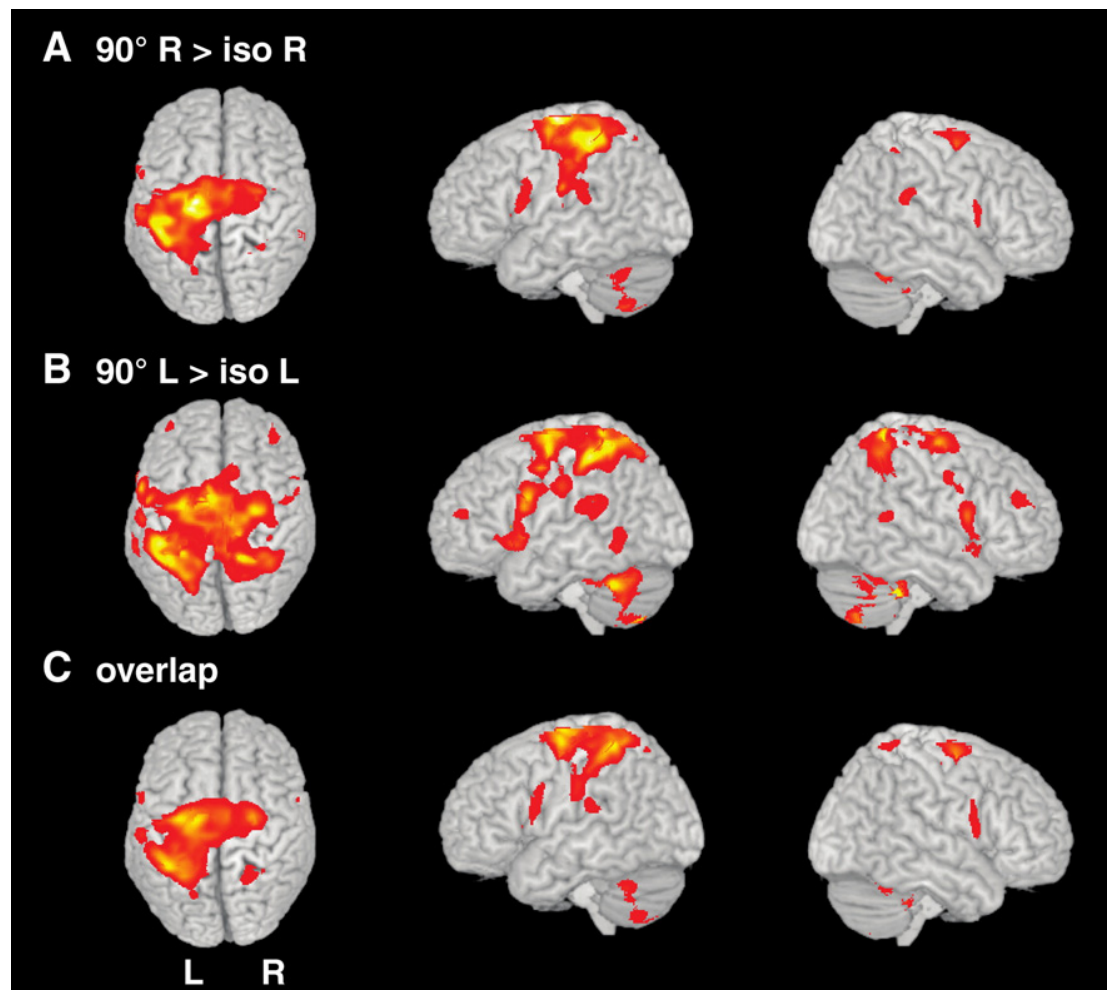

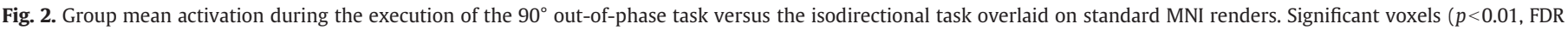

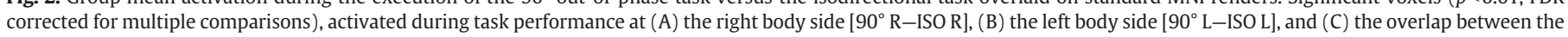
two conditions [ $\left(90^{\circ} \mathrm{r}-\right.$ ISO $\left.\mathrm{r}\right) \cap\left(90^{\circ} \mathrm{l}-\right.$ ISO l)], are indicated. L, left hemisphere; R, right hemisphere. 
Table 3

Significant activations resulting from the conjunction analysis $\left(90^{\circ} \mathrm{r}-\mathrm{ISO} \mathrm{r}\right) \cap\left(90^{\circ} \mathrm{l}-\mathrm{ISO} \mathrm{l}\right)$.

\begin{tabular}{|c|c|c|c|c|c|}
\hline \multirow[t]{2}{*}{ Brain region } & \multirow[t]{2}{*}{ Hemisphere } & \multicolumn{3}{|c|}{ Coordinates } & \multirow[t]{2}{*}{$t$-value } \\
\hline & & $x$ & $y$ & $z$ & \\
\hline \multirow[t]{2}{*}{ Superior frontal gyrus (SMA) } & $\mathrm{L}$ & -14 & -6 & 60 & 5.84 \\
\hline & $\mathrm{R}$ & 6 & -8 & 64 & 5.67 \\
\hline Superior frontal gyrus (PMd) & $\mathrm{R}$ & 28 & -10 & 58 & 6.78 \\
\hline Superior frontal sulcus (PMd) & $\mathrm{L}$ & -30 & -8 & 62 & 7.47 \\
\hline \multirow[t]{2}{*}{ Inferior frontal gyrus pars opercularis } & $\mathrm{L}$ & -56 & 8 & 18 & 4.18 \\
\hline & $\mathrm{R}$ & 54 & 8 & 12 & 3.85 \\
\hline Precentral gyrus (PMd) & $\mathrm{L}$ & -18 & -10 & 72 & 7.41 \\
\hline \multirow[t]{2}{*}{ Inferior precentral sulcus (PMv) } & $\mathrm{L}$ & -58 & 6 & 26 & 4.25 \\
\hline & $\mathrm{R}$ & 56 & 6 & 22 & 3.54 \\
\hline Central sulcus & $\mathrm{L}$ & -14 & -38 & 74 & 4.94 \\
\hline Postcentral gyrus (S1 hand area) & $\mathrm{L}$ & -48 & -30 & 54 & 7.52 \\
\hline Inferior postcentral sulcus & $\mathrm{L}$ & -58 & -20 & 32 & 4.66 \\
\hline Paracentral lobule (S1 foot area) & $\mathrm{L}$ & -2 & -22 & 64 & 7.16 \\
\hline \multirow[t]{2}{*}{ Superior parietal gyrus } & $\mathrm{L}$ & -26 & -44 & 70 & 7.64 \\
\hline & $\mathrm{R}$ & 24 & -48 & 70 & 4.17 \\
\hline Supramarginal gyrus & $\mathrm{L}$ & -58 & -28 & 22 & 3.63 \\
\hline Posterior intraparietal sulcus & $\mathrm{R}$ & 32 & -54 & 42 & 3.82 \\
\hline \multicolumn{5}{|l|}{ postcentral sulcus } & 7.83 \\
\hline Insula & $\mathrm{L}$ & -32 & 18 & 8 & 4.11 \\
\hline Putamen & $\mathrm{L}$ & -22 & 2 & 6 & 3.82 \\
\hline Thalamus & $\mathrm{L}$ & -22 & -18 & 4 & 3.75 \\
\hline Cerebellar vermis (IV) & $\mathrm{R}$ & 10 & -52 & -24 & 5.97 \\
\hline Cerebellar vermis (V) & $\mathrm{R}$ & 4 & -56 & -18 & 6.26 \\
\hline Cerebellar hemisphere (VI) & $\mathrm{R}$ & 24 & -54 & -30 & 6.37 \\
\hline \multirow[t]{2}{*}{ Cerebellar hemisphere (VIII A) } & $\mathrm{L}$ & -30 & -62 & -52 & 4.36 \\
\hline & $\mathrm{R}$ & 24 & -60 & -52 & 5.05 \\
\hline Cerebellar hemisphere $(\mathrm{Cr} \mathrm{I})$ & $\mathrm{L}$ & -38 & -52 & -34 & 5.05 \\
\hline
\end{tabular}

$t$ values and localizations (MNI coordinates) of activation peaks showing significant activations ( $p<.01$, FDR corrected) resulting from the following conjunction: $\left(90^{\circ} \mathrm{r}-\mathrm{ISO} \mathrm{r}\right) \cap$ ( $90^{\circ}$ l-ISO l). L, left hemisphere; R, right hemisphere.

\section{Discussion}

In searching for the neural basis of abstract movement representations, we investigated which brain areas are commonly activated when a newly acquired coordination task is performed with the right versus left body side. A three-step procedure was used to identify the areas that were uniquely related to new coordination learning as well as commonly activated across the left and right interlimb coordination tasks. A parieto-(pre)motor network was identified with more prominent activity in the left than right hemisphere.

Below, we will primarily address the cortical and subcortical areas that are shared between movements of both body sides, presumably constituting candidate loci for abstract encoding of movement. These areas will be linked with those identified during action observation of complex multilimb movements to further amplify their assumed role in abstract representations. Even though we cannot exclude the possibility that some of the obtained activations might have emerged as a result of increased attention/effort required for the $90^{\circ}$ out-ofphase as compared to the ISO task, substantial practice was provided prior to scanning to overcome this problem. The kinematic analysis revealed that automaticity was obtained by the fifth day of practice, underscoring the decreasing role of effort/attention to mediate performance at this stage.

Brain activations common to the learned left and right $90^{\circ}$ out-of-phase coordination mode: in search for the abstract motor representation

The conjunction of the brain areas associated with the left and right $90^{\circ}$ out-of-phase pattern revealed a bilateral network with activations being much more prominent in the left than right hemisphere. The areas thus obtained were considered candidate loci for representing the effector-independent neural representation of the complex coordination skill. Whereas activation in these areas may also be associated with movement complexity, this argument is less persuasive because extensive practice was provided to acquire the task. Cortical activations included bilateral premotor areas (PMd, PMv and SMA), bilateral IFGpo and left insula, left primary sensorimotor cortex (hand and foot area), bilateral superior parietal lobe extending to the left intraparietal sulcus, and inferior parietal lobe (supramarginal and angular gyrus). Subcortical activations were obtained in the left putamen and thalamus, and (primarily) right cerebellum (anterior and posterior). Because of their prominent involvement, we will focus on the parietal-(pre)motor network as well as the cerebellar activations, as discussed next.

\section{Cortical areas shared between left and right limb coordination tasks}

The present findings speak to Liepmann's (1905) seminal observations in apraxia patients: (a) lateralization of a skilled movement representation to the left hemisphere and (b) identification of the premotor-parietal complex as the principal candidate network involved in the formation of abstract representations. Previous data from TMS, brain imaging and lesion experiments, have underscored the predominance of left lateralized activation for motor skills. Functional imaging studies have provided evidence of left hemisphere dominance when comparing right and left hand performance (Dassonville et al., 1998; Johnson-Frey et al., 2005; Serrien et al., 2003; Verstynen et al. 2007). Following left hemisphere damage, contralateral as well as ipsilateral motor deficits are commonly found, especially when the task is cognitively demanding (Haaland and Harrington, 1996), such as during sequencing (Haaland and Harrington, 1994) or bimanual skills (Wyke, 1971). Conversely, right hemisphere damage is more prone to producing contralateral deficits (Haaland and Harrington, 1994).

Liepmann suggested that the left parietal cortex is the critical site for skilled motor representations, projecting to the bilateral premotor areas through intrahemispheric and transcallosal pathways (Liepmann, 1905). Our study supported this general contention but provided new (more detailed) information, i.e., we obtained activation in superior parietal lobe extending into the left intraparietal sulcus as well as inferior parietal lobe.

The role of the parietal cortex in multilimb coordination has been demonstrated repeatedly (de Jong et al., 2002; Ullen et al., 2003; Wenderoth et al., 2004; Debaere et al., 2004a; Heuninckx et al., 2005, 2008; Serrien et al., 2001). The superior parietal gyrus is known to be involved in sensorimotor integration and online processing of somatosensory information from multiple limbs for movement guidance, i.e. linking sensory signals and motor commands (Rizzolatti et al., 1997; Iwamura, 1998). Furthermore, (a) IPS is considered a critical node in the higher order dynamic control of action, including the representation of intended action goals. In a review of grasping studies, Tunik et al. (2007) proposed that this site may represent a diverse range of goal oriented actions (mean $X, Y, Z=-39.7,-39.3$, 43.2). In our study, the peak location was more superior $(-38,-44$, 62 ), closely resembling the coordinates obtained in action observation studies on complex dancing skills, i.e., $-33,-45,54$ (Calvo-Merino et al., 2005) and $-33,-48$, and 66 (Cross et al., 2006). This similarity between action execution during ipsilateral coordination and action observation during dancing (both involving complex interlimb coordination) underscores the abstract nature of SPL/IPS representations for complex action goals and suggests that this area is of focal importance for encoding the complex spatiotemporal organization among limb movements that characterizes interlimb coordination (i.e., knowing how to perform the action). Damage to the left parietal cortex gives rise to a wide range of bilateral motor deficits including 
A $z=0$

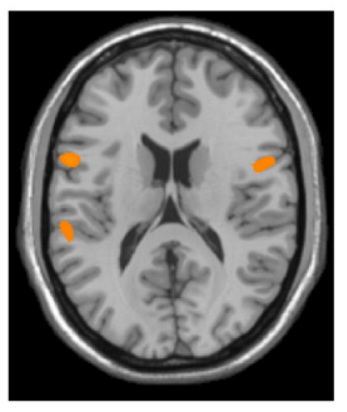

D $z=53$

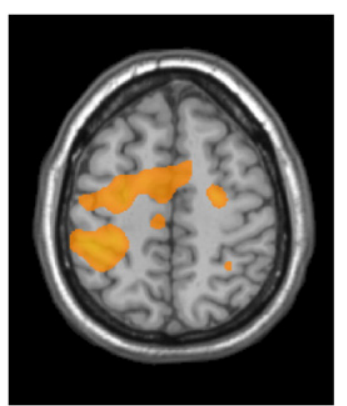

$\mathbf{F} \mathrm{x}=5$

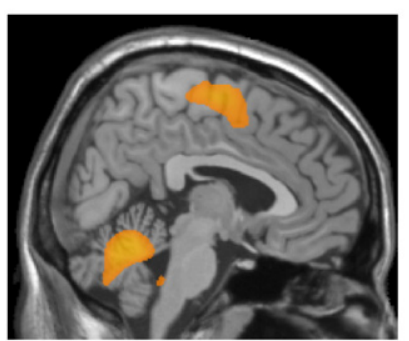

B $z=6$

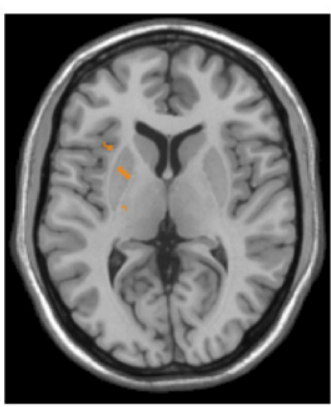

$E z=63$

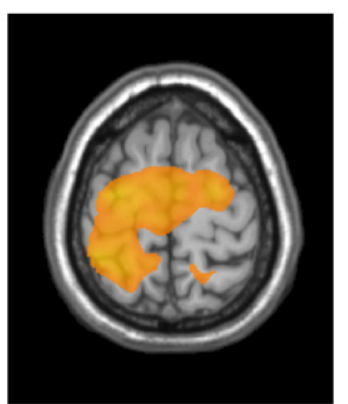

G $y=5$

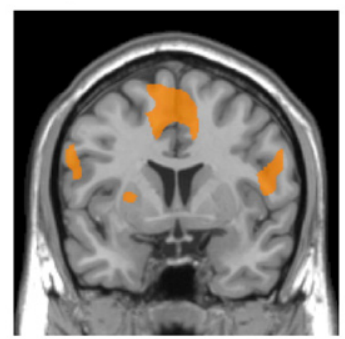

C z $=44$

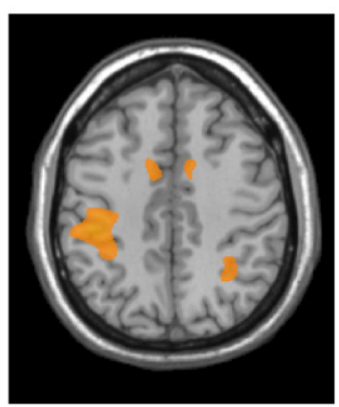

H $y=-62$

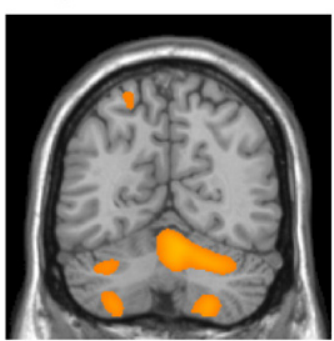

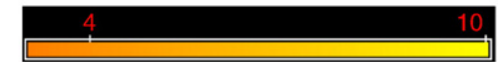

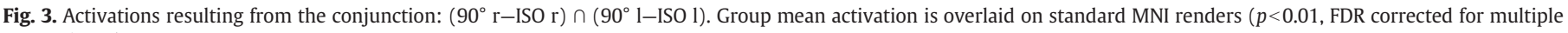
comparisons).

ideomotor apraxia (Haaland et al., 2000; Serrien et al., 2001) and poor interjoint coordination (Poizner et al., 1995).

With respect to the inferior parietal lobe, we observed activity in the left supramarginal gyrus $(-58,-28,22)$. This left IPL activation is adjacent to that obtained in a group of dancers who observed and judged familiar (practiced) and unfamiliar (unpracticed) dance routines $(-57,-27,36)$. Activity in IPL was greatest when participants simulated actions they had practiced themselves and judged they could perform well. Together with IFG, IPL is considered part of the human mirror system (Rizzolatti and Craighero, 2004). IPL is often activated when humans observe, prepare or simulate limb actions.

In addition to the aforementioned (primarily left) parietal activations, an activation spot in right IPS $(32,-54,42)$ was observed without a significant left counterpart. In a study using delayed feedback techniques, Farrer and coworkers (2008) showed that a neighboring region $(44,-54,38)$ was associated with discrepancy between intended and actual movement consequences. Additionally, Mars et al. (2007) found posterior right IPS activation in a locus very similar to ours $(32,-54,44)$ that they associated with programming of a motor response in the context of an already existing motor program. The latter account is meaningful for the present coordination tasks because the continuous comparison between intended and actual sensory consequences may result in online action adjustments and movement replanning. As such, this activation is not considered being part of the abstract movement representation.

Even though the premotor and motor cortex exhibit a certain level of effector-driven somatotopy, our data suggest that these areas may nevertheless host effector-independent representations. We found evidence for activations in bilateral premotor/motor areas with more prominent activity in the left than right hemisphere: SMA, PMd, PMv, and IFG pars opercularis. Interestingly, IFGpo (BA44) activation is often associated with monkey Area F5 in which mirror neurons have been observed (Rizzolatti and Craighero, 2004). The activations in our study $(-56,8,18 ; 54,8,12)$ were similar to those obtained in observation of dancing skills $(-48,12,21 ;-54,6,33)$ (Cross et al., 2006). Furthermore, this area has been further subdivided into a dorsal and ventral part, with the former sector being activated during action observation and imitation (coordinates close to ours, +-48 to 56,8 to 18,16 to 24 ) (Molnar-Szakacs et al., 2005). Our locus is also close to an activation peak $(-50,-2,12)$ that has shown mirror neuron properties, i.e., activation during execution as well as observation of grasping actions (Kilner et al., 2009). The converging IFGpo activation during actual motor coordination as well as during observation of simple and complex gross motor skills suggests this 
area as a potential locus for representing abstract movement codes. Further confirmation from studies combining action execution and observation is however mandatory.

In addition, PMv, PMd, and SMA activity was also observed. The extensive premotor activations exhibited a bilateral signature. This may be related to various factors including degree of skill complexity, the use of foot movements, or combinations thereof. Indeed, previous studies have shown lower degrees of activity lateralization for foot as compared to hand movements (Kapreli et al., 2006; Lafleur et al., 2002; Sahyoun et al., 2004). Such activations may compromise the search for abstract movement representations. However, their role in abstract movement representations is also conceivable. Indeed, limbindependent activations have been documented for left dorsal premotor cortex in the context of bilateral hand rotation (Viviani et al., 1998), choice reaction time tasks (Schluter et al., 2001) and finger-to-thumb sequencing tasks (Porro et al., 2000; Hlustik et al., 2002). Left premotor effector-independent activation has also been observed during imagery of a simple finger compressing task with the right or left hand (Kuhtz-Buschbeck et al., 2003). It has been suggested that the left PMd plays a fundamental role in the selection and retrieval of abstract action plans from the parietal lobe (Harrington et al., 2000) and the translation of these motor programs into spatial coordinates and plans for execution (Sakai et al., 1998; Thoenissen et al., 2002). Furthermore, PMd, as well as SMA, is known to contribute to the preparation and organization of forthcoming movements that are rehearsed from memory (Gerloff et al., 1997). The left PMd is also involved in processes related to the abstract, temporal aspects of a task (Halsband et al., 1993; van Mier et al., 1998). When coordinating the hand and foot in a $90^{\circ}$ out-of-phase mode, precise timing is required to successively reach the turning points during the cyclical movements of both limbs, in time with the paced rhythm and while obeying rapidly changing spatial (directional) interlimb relations.

More generally, the role of the premotor-parietal network in movement representations has also been supported in studies specifically addressing the neural substrate of effector-independent motor representations without a scope on motor lateralization. Comparing signing with the right finger or toe (acquired skill) versus finger/toe zigzagging (intrinsic skill), Rijntjes et al. (1999) observed that signing with the toe activated areas belonging to the right finger representation in the left intraparietal sulcus and the SMA. Moreover, limb-independent activation was also revealed in the dorsal as well as ventral premotor regions. Sustained activation in the left inferior parietal cortex (BA 40) was also demonstrated after transfer of an implicit sequence learning task from the right fingers to the right arm, suggesting that sequence knowledge is represented at the abstract response goal level (Grafton et al., 1998).

\section{Subcortical areas shared between left and right limb coordination tasks}

The conjunction analysis revealed that subcortical activations were shared between left and right limb coordination patterns, notably in the left basal ganglia (putamen), left thalamus, and (predominantly) right cerebellum. The cerebellar activations are consistent with the existence of two body representations in the cerebellum, one in the anterior $(\mathrm{V}, \mathrm{VI})$ and a second in the posterior lobe (VIIIA/B) (Stoodley and Schmahmann, 2009). Previous work on the acquisition of new bimanual coordination patterns has already underscored the important role of the basal ganglia and cerebellum in motor automaticity (Debaere et al., 2004b; Puttemans et al., 2005). The present findings are also consistent with a role of the cerebellum in the temporal organization of coordinated action (Miall et al., 2001; Thach et al., 1992). Cerebellar damage shows more severe impairment in multijoint than single joint movements (Goodkin and Thach, 2003). With respect to forward models of motor control, we consider both the parietal cortex and cerebellum as potential nodes for relating actual with predicted sensory consequences. In this context, it is meaningful to consider a cerebellar (implicit) route for fast processing and a parietal (explicit) route for monitoring intention and motor plans at higher levels (Sirigu et al., 2004).

The more important question that has received very little attention in the literature is whether subcortical structures should be considered in the context of effector-independent representations. Since Liepmann's (1905) seminal work, abstract action goal representations have traditionally been assigned to higher cortical sites. However, the multiple sites in the right anterior and posterior cerebellum prompt questions about their possible role in abstract motor representations. We will approach this issue from the perspective of control theory as well as neuroanatomy (exploring evidence for bilateral limb control from unilateral cerebellar sites).

Skill learning not only implies the generation of new motor command structures but also of models representing the (expected) sensory consequences associated with planned movement (Schmidt, 1975). More recent versions of this notion refer to forward internal models that predict the sensorimotor consequences of actions and the future state of the moving limb. This permits fast adjustments to the unfolding movement and reduces dependence on slower feedback control. Some studies suggest that the cerebellum provides the predictive internal models necessary for the coordination between eye and hand movements (e.g., Miall and Jenkinson, 2005; Miall et al., 2001; Roitman et al., 2005; Wolpert et al., 1998). Such internal models may appear effector specific at first sight because these neural representations of correct input-output mappings are built up through error feedback in specific contexts. However, to the extent that general rules are learned and applied, such forward models may also contain a more abstract signature. More research is clearly required to further explore this hypothesis.

From an anatomical perspective, unilateral activity in the cerebellum affects movements on both sides of the body under certain conditions. Recent single-cell recording studies in monkeys suggest that neurons in the lateral cerebellar cortex code for "abstract" movement parameters, irrespective of the effector (Gregor et al., 2004). This suggests that at least some cerebellar neurons are coding according to an effector-independent scheme. Furthermore, the deep cerebellar nuclei have access to limb muscles on both sides of the body (Soteropoulos and Baker, 2008). In addition, unilateral cerebellar lesions in humans influence movements bilaterally (Immisch et al., 2003). Inputs from the cortex to the cerebellum may be partly bilateral, as observed in a small percentage of the pontine neurons (Brodal, 1980; Rosina and Provini, 1981). All together, there is some credibility to the notion that the cerebellum may be involved in coding abstract (effector-independent) features of movement and that unilateral areas exert bilateral control. However, these preliminary ideas await further confirmation.

\section{Summary}

In interlimb coordination tasks, a parieto-frontal network with predominant activity in the left hemisphere assumes a prominent role in effector-independent encoding of movement. More specifically, the left parietal cortex, particularly the superior parietal lobule may be a critical node for action representation at the most abstract level of the hierarchy, sending its output to (bilateral) premotor areas. It is hypothesized that the parietal cortex stores the most abstract action goals, more specifically the spatiotemporal relationships between effectors involved in coordination. It represents the highest level in the hierarchy of action representations. Among the premotor areas, the IFGpo is a noteworthy candidate endowed with mirror neuron properties because it does not only become active during performance but also during expert observation of complex coordination skills, underscoring its role in abstract effector-independent encoding of complex movement. Overall, it is proposed that a left-hemisphere parieto-premotor complex represents the neural correlate of motor 
equivalence, a hallmark of central nervous system flexibility in reaching action goals through various means, including intransitive actions such as those studied here.

\section{Acknowledgments}

Support for this study was provided via OT and IDO grants from the Research Council of K.U. Leuven, Belgium (Contract OT/07/073 and IDO/06/004), the Flanders Fund for Scientific Research (Project G.0593.08), Grant P6/29 from the Interuniversity Attraction Poles program of the Belgian federal government, and Impulse Grant IMPH/06/GHW.

\section{References}

Baldissera, F., Cavallari, P., Civaschi, P., 1982. Preferential coupling between voluntary movements of ipsilateral limbs. Neurosci. Lett. 34, 95-100.

Brodal, P., 1980. The cortical projection to the nucleus reticularis tegmenti pontis in the rhesus monkey. Exp. Brain Res. 38, 19-27.

Calvo-Merino, B., Glaser, D.E., Grezes, J., Passingham, R.E., Haggard, P., 2005. Action observation and acquired motor skills: an fMRI study with expert dancers. Cereb. Cortex 15, 1243-1249.

Carson, R.G., Goodman, D., Kelso, J.A., Elliott, D., 1995. Phase transitions and critical fluctuations in rhythmic coordination of ipsilateral hand and foot. J. Mot. Behav. 27, $11-224$.

Criscimagna-Hemminger, S.E., Donchin, O., Gazzaniga, M.S., Shadmehr, R., 2003. Learned dynamics of reaching movements generalize from dominant to nondominant arm. J. Neurophysiol. 89, 168-176.

Cross, E.S., Hamilton, A.F.C., Grafton, S.T., 2006. Building a motor simulation de novo: observation of dance by dancers. NeuroImage 31, 1257-1267.

Dassonville, P., Lewis, S.M., Zhu, X.H., Ugurbil, K., Kim, S.G., Ashe, J., 1998. Effects of movement predictability on cortical motor activation. Neurosci. Res. 32, 65-74.

de Jong, B.M., Leenders, K.L., Paans, A.M., 2002. Right parieto-premotor activation related to limb-independent antiphase movement. Cereb.Cortex 12,1213-1217.

Debaere, F., Wenderoth, N., Sunaert, S., Van Hecke, P., Swinnen, S.P., 2004a. Changes in brain activation during the acquisition of a new bimanual coordination task. Neuropsychologia 42, 855-867.

Debaere, F., Wenderoth, N., Sunaert, S., Van Hecke, P., Swinnen, S.P., 2004b. Changes in brain activation during the acquisition of a new bimanual coordination task Neuropsychologia 42, 855-867.

Farrer, C., Frey, S.H., Van Horn, J.D., Tunik, E., Turk, D., Inati, S., Grafton, S.T., 2008. The angular gyrus computes action awareness representations. Cereb. Cortex 18 254-261.

Genovese, C.R., Lazar, N.A., Nichols, T., 2002. Thresholding of statistical maps in functional neuroimaging using the false discovery rate. NeuroImage 15, 870-878.

Gerloff, C., Corwell, B., Chen, R., Hallett, M., Cohen, L.G., 1997. Stimulation over the human supplementary motor area interferes with the organization of future elements in complex motor sequences. Brain 120, 1587-1602.

Goodkin, H.P., Thach, W.T., 2003. Cerebellar control of constrained and unconstrained movements. I. Nuclear inactivation. J. Neurophys. 89, 884-895.

Grafton, S.T., Hazeltine, E., Ivry, R.B., 1998. Abstract and effector-specific representations of motor sequences identified with PET. J. Neurosci. 18, 9420-9428.

Gregor, B., Norris, S.A., Thach, W.T., 2004. Spike firing in the lateral cerebellar cortex correlated with movement and motor parameters irrespective of the effector limb. J. Neurophysiol. 91, 576-582.

Haaland, K.Y., Harrington, D.L., 1994. Limb-sequencing deficits after left but not right hemisphere damage. Brain Cogn. 24, 104-122.

Haaland, K.Y., Harrington, D.L., 1996. Hemispheric asymmetry of movement. Curr. Opin. Neurobiol. 6, 796-800.

Haaland, K.Y., Harrington, D.L., Knight, R.T., 2000. Neural representations of skilled movement. Brain 123, 2306-2313.

Haaland, K.Y., Elsinger, C.L., Mayer, A.R., Durgerian, S., Rao, S.M., 2004. Motor sequence complexity and performing hand produce differential patterns of hemispheric lateralization. J.Cogn. Neurosci. 16, 621-636.

Halsband, U., Ito, N., Tanji, J., Freund, H.J., 1993. The role of premotor cortex and the supplementary motor area in the temporal control of movement in man. Brain 116, 243-266.

Harrington, D.L., Rao, S.M., Haaland, K.Y., Bobholz, J.A., Mayer, A.R., Binderx, J.R., Cox, R.W., 2000. Specialized neural systems underlying representations of sequential movements. J. Cogn. Neurosci. 12, 56-77.

Heuninckx, S., Wenderoth, N., Swinnen, S.P., 2005. Neural basis of aging: the penetration of cognition into action control. J. Neurosci. 25, 6787-6796.

Hlustik, P., Solodkin, A., Gullapalli, R.P., Noll, D.C., Small, S.L., 2002. Functional lateralization of the human premotor cortex during sequential movements. Brain Cogn. 49, 54-62.

Imamizu, H., Shimojo, S., 1995. The locus of visual-motor learning at the task or manipulator level: implications from intermanual transfer. J. Exp. Psychol. Hum. Percept. Perform. 21, 719-733.

Immisch, I., Quinten, J., Straube, A., 2003. Unilateral cerebellar lesions influence arm movements bilaterally. NeuroReport $14,837-840$
Iwamura, Y., 1998. Hierarchical somatosensory processing. Curr. Opin. Neurobiol. 8, $522-528$

Johnson-Frey, S.H., Newman-Norlund, R., Grafton, S.T., 2005. A distributed left hemisphere network active during planning of everyday tool use skills. Cereb. Cortex 15, 681-695.

Kapreli, E., Athanasopoulos, S., Papathanasiou, M., Van Hecke, P., Strimpakos, N., Gouliamos, A., Peeters, R., Sunaert, S., 2006. Lateralization of brain activity during lower limb joints movement. An fMRI study. NeuroImage 32, 1709-1721.

Kelso, J.A.S., Scholz, J.P., Schöner, G., 1986. Non-equilibrium phase transitions in coordinated biological motions: critical fluctuations. Phys. Lett. A 118, 279-284.

Kilner, J.M., Neal, A., Weiskopf, N., Friston, K.J., Frith, C.D., 2009. Evidence of mirror neurons in human inferior frontal gyrus. J. Neurosci. 29, 10153-10159.

Kuhtz-Buschbeck, J.P., Mahnkopf, C., Holzknecht, C., Siebner, H., Ulmer, S., Jansen, O., 2003. Effector-independent representations of simple and complex imagined finger movements: a combined fMRI and TMS study. Eur. J. Neurosci. 18, 3375-3387.

Lafleur, M.F., Jackson, P.L., Malouin, F., Richards, C.L., Evans, A.C., Doyon, J., 2002. Motor learning produces parallel dynamic functional changes during the execution and imagination of sequential foot movements. Neurolmage 16, 142-157.

Lashley, K.S., 1930. Basic neural mechanisms in behavior. Psychol. Rev. 37, 1-24.

Lee, T.D., Swinnen, S.P., Verschueren, S., 1995. Relative phase alterations during bimanual skill acquisition. J. Mot. Behav. 27, 263-274.

Liepmann, H., 1905. Die linke Hemisphare und das Handeln. Munch. Med. Wochenschr. 49 2322-2326-2375-2378.

Mars, R.B., Piekema, C., Coles, M.G.H., Hulstijn, W., Toni, I., 2007. On the programming and reprogramming of actions. Cereb. Cortex 17, 2972-2979.

Miall, R.C., Jenkinson, E.W., 2005. Functional imaging of changes in cerebellar activity related to learning during a novel eye-hand tracking task. Exp. Brain Res. 166, 170-183.

Miall, R.C., Reckess, G.Z., Imamizu, H., 2001. The cerebellum coordinates eye and hand tracking movements. Nat. Neurosci. 4, 638-644.

Molnar-Szakacs, I., Iacoboni, M., Koski, L., Mazziotta, J.C., 2005. Functional segregation within pars opercularis of the inferior frontal gyrus: evidence from fMRI studies of imitation and action observation. Cereb. Cortex 15, 986-994.

Nichols, T., Brett, M., Andersson, J., Wager, T., Poline, J.B., 2005. Valid conjunction inference with the minimum statistic. Neurolmage 25, 653-660.

Oldfield, R.C., 1971. The assessment and analysis of handedness: the Edinburgh inventory. Neuropsychologia 9, 97-113.

Poizner, H., Clark, M.A., Merians, A.S., Macauley, B., Gonzalez Rothi, L.J., Heilman, K.M., 1995. Joint coordination deficits in limb apraxia. Brain 118, 227-242.

Porro, C.A., Cettolo, V., Francescato, M.P., Baraldi, P., 2000. Ipsilateral involvement of primary motor cortex during motor imagery. Eur. J. Neurosci. 12, 3059-3063.

Puttemans, V., Wenderoth, N., Swinnen, S.P., 2005. Changes in brain activation during the acquisition of a multifrequency bimanual coordination task: from the cognitive stage to advanced levels of automaticity. J. Neurosci. 25, 4270-4278.

Rijntjes, M., Dettmers, C., Büchel, C., Kiebel, S., Frackowiak, R.C., Weiller, C., 1999. A blueprint for movement: functional and anatomical representations in the human motor system. J. Neurosci. 19, 8043-8048.

Rizzolatti, G., Craighero, L., 2004. The mirror neuron system. Ann. Rev. Neurosci. 27, 169-192.

Rizzolatti, G., Fogassi, L., Gallese, V., 1997. Parietal cortex: from sight to action. Curr. Opin. Neurobiol. 7, 562-567.

Roitman, A.V., Pasalar, S., Johnson, M.T.V., Ebner, T.J., 2005. Position, direction of movement, and speed tuning of cerebellar Purkinje cells during circular manual tracking in monkey. J. Neurosci. 25, 9244-9257.

Rosina, A., Provini, L., 1981. Pontine projections to crus I and crus II of the cat cerebellum. A horseradish peroxidase study. Neuroscience 6, 2613-2624.

Sahyoun, C., Floyer-Lea, A., Johansen-Berg, H., Matthews, P.M., 2004. Towards an understanding of gait control: brain activation during the anticipation, preparation and execution of foot movements. NeuroImage 21, 568-575.

Sakai, K., Hikosaka, O., Miyauchi, S., Takino, R., Sasaki, Y., Putz, B., 1998. Transition of brain activation from frontal to parietal areas in visuomotor sequence learning. J. Neurosci. 18, 1827-1840.

Schluter, N.D., Krams, M., Rushworth, M.F., Passingham, R.E., 2001. Cerebral dominance for action in the human brain: the selection of actions. Neuropsychologia 39, $105-113$.

Schmidt, R.A., 1975. A schema theory of discrete motor skill learning. Psychol. Rev. 82, 225-260.

Serrien, D.J., Nirkko, A.C., Lovblad, K.O., Wiesendanger, M., 2001. Damage to the parietal lobe impairs bimanual coordination. NeuroReport 12, 2721-2724.

Serrien, D.J., Cassidy, M.J., Brown, P., 2003. The importance of the dominant hemisphere in the organization of bimanual movements. Hum. Brain Mapp. 18, 296-305.

Sirigu, A., Daprati, E., Ciancia, S., Giraux, P., Nighoghossian, N., Posada, A., Haggard, P., 2004. Altered awareness of voluntary action after damage to the parietal cortex. Nat. Neurosci. 7, 80-84

Soteropoulos, D., Baker, S.N., 2008. Bilateral representation in the deep cerebellar nuclei. J. Physiol. 586, 1117-1136.

Stoodley, C.J., Schmahmann, J.D., 2009. Functional topography in the human cerebellum: a meta-analysis of neuroimaging studies. Neurolmage 44, 489-501.

Swinnen, S.P., 2002. Intermanual coordination: from behavioural principles to neuralnetwork interactions. Nat. Rev. Neurosci. 3, 350-361.

Swinnen, S.P., Dounskaia, N., Verschueren, S., Serrien, D.J., Daelman, A., 1995. Relative phase destabilization during interlimb coordination: the disruptive role of kinesthetic afferences induced by passive movement. Exp. Brain Res. 105, 439-454. 
Swinnen, S.P., Dounskaia, N., Walter, C.B., Serrien, D.J., 1997a. Preferred and induced coordination modes during the acquisition of bimanual movements with a 2:1 frequency ratio. J. Exp. Psychol. Hum. Percept. Perform. 23, 1087-1110.

Swinnen, S., Jardin, K., Meulenbroek, R., Dounskaia, N., Hofkens-Van Den Brandt, M., 1997b. Egocentric and allocentric constraints in the expression of patterns of interlimb coordination. J. Cogn. Neurosci. 9, 348-377.

Talairach, J., Tournoux, P., 1998. Co-planar stereotaxic atlas of the human brain. Thieme, New York.

Thach, W.T., Goodkin, H.P., Keating, J.G., 1992. The cerebellum and the adaptive coordination of movement. Annu. Rev. Neurosci. 15, 403-442.

Thoenissen, D., Zilles, K., Toni, I., 2002. Differential involvement of parietal and precentral regions in movement preparation and motor intention. J. Neurosci. 22, 9024-9034.

Tunik, E., Rice, N.J., Hamilton, A., Grafton, S.T., 2007. Beyond grasping: representation of action in human anterior intraparietal sulcus. NeuroImage 36 (Suppl. 2), T77-T86.

Ullen, F., Forssberg, H., Ehrsson, H.H., 2003. Neural networks for the coordination of the hands in time. J. Neurophysiol. 89, 1126-1135.

van Mier, H., Tempel, L.W., Perlmutter, J.S., Raichle, M.E., Petersen, S.E., 1998. Changes in brain activity during motor learning measured with PET: effects of hand of performance and practice. J. Neurophysiol. 80, 2177-2199.

Vangheluwe, S., Puttemans, V., Wenderoth, N., Van Baelen, M., Swinnen, S.P., 2004. Inter- and intralimb transfer of a bimanual task: generalisability of limb dissociation. Behav. Brain Res. 154, 535-547.
Vangheluwe, S., Wenderoth, N., Swinnen, S.P., 2005. Learning and transfer of an ipsilateral coordination task: evidence for a dual-layer movement representation. J. Cogn. Neurosci. 17, 1460-1470.

Verstynen, T., Diedrichsen, J., Albert, N., Aparicio, P., Ivry, R.B., 2007. Ipsilateral motor cortex activity during unimanual hand movements relates to task complexity. J. Neurophysiol. 93, 1209-1222.

Viviani, P., Perani, D., Grassi, F., Bettinardi, V., Fazio, F., 1998. Hemispheric asymmetries and bimanual asynchrony in left- and right-handers. Exp. Brain Res. 120, 531-536.

Wenderoth, N., Debaere, F., Sunaert, S., Van Hecke, P., Swinnen, S.P., 2004. Parietopremotor areas mediate directional interference during bimanual movements. Cereb. Cortex 14, 1153-1163.

Wolpert, D.M., Miall, R.C., Kawato, M., 1998. Internal models in the cerebellum. Trends Cogn. Sci. 2, 338-347.

Wright, C, 1990. Generalized motor programs: reexamining claims of effector independence in writing. In: Jeannerod, M. (Ed.), Attention and performance XIII: motor representation and control. Erlbaum Associates, Hillsdale pp. $294-320$.

Wyke, M., 1971. The effects of brain lesions on the learning performance of a bimanual co-ordination task. Cortex 7, 59-72.

Zanone, P.G., Kelso, J.A., 1992. Evolution of behavioral attractors with learning: nonequilibrium phase transitions. J. Exp. Psychol. Hum. Percept. Perform. 18, 403-421. 